Pacific

Journal of

Mathematics

ASYMPTOTICALLY LINEAR HAMILTONIAN SYSTEMS WITH LAGRANGIAN BOUNDARY CONDITIONS

Chun-Gen LiU 


\title{
ASYMPTOTICALLY LINEAR HAMILTONIAN SYSTEMS WITH LAGRANGIAN BOUNDARY CONDITIONS
}

\author{
CHUN-Gen LiU
}

\begin{abstract}
We study the multiplicity of the solutions of certain asymptotically linear Hamiltonian systems with a Lagrangian boundary condition.
\end{abstract}

\section{Introduction and main results}

We consider the solutions of the nonlinear Hamiltonian systems with Lagrangian boundary condition

$$
\dot{x}(t)=J H^{\prime}(t, x(t)), \quad x(0) \in L, \quad x(1) \in L .
$$

where $x(t) \in \mathbb{R}^{2 n}$ and

$$
J=\left(\begin{array}{cc}
0 & -I_{n} \\
I_{n} & 0
\end{array}\right)
$$

is the standard symplectic matrix with $I_{n}$ the identity in $\mathbb{R}^{n}$, and $L \in \Lambda(n)$, where $\Lambda(n)$ is the set of all Lagrangian subspaces of $\left(\mathbb{R}^{2 n}, \omega_{0}\right)$ with standard symplectic form $\omega_{0}=\sum_{j=1}^{n} d x_{j} \wedge d y_{j}$. The Hamiltonian function $H \in C^{2}\left([0,1] \times \mathbb{R}^{2 n}, \mathbb{R}\right)$ satisfies these conditions:

- $\left(H_{0}\right): H^{\prime}(t, 0) \equiv 0, t \in[0,1]$.

- $\left(H_{\infty}\right)$ : There exist continuous symmetric matrix functions $B_{1}(t)$ and $B_{2}(t)$ with $i_{L}\left(B_{1}\right)=i_{L}\left(B_{2}\right), v_{L}\left(B_{2}\right)=0$ such that

$$
B_{1}(t) \leq H^{\prime \prime}(t, x) \leq B_{2}(t)
$$

for all $(t, x)$ with $|x| \geq r$ for some large $r>0$ and for all $t \in[0,1]$.

For two symmetric matrices $A$ and $B, A \geq B$ means that $A-B$ is a semipositive definite matrix, and $A>B$ similarly means that $A-B$ is a positive definite matrix.

For a Lagrangian subspace $L$ of the standard symplectic vector space $\left(\mathbb{R}^{2 n}, \omega_{0}\right)$, [Liu 2007] defined the Maslov-type index pair $\left(i_{L}(B), v_{L}(B)\right) \in \mathbb{Z} \times\{0,1, \ldots, n\}$ for a continuous symmetric matrix function $B:[0,1] \rightarrow \mathrm{L}_{s}(2 n)$ (here $\mathrm{L}_{s}(2 n)$ is the

MSC2000: 58E05, 34C25.

Keywords: index theory, Hamiltonian systems, Lagrangian boundary conditions.

Partially supported by the National Natural Science Foundation of China, 973 program of MOST. 
set of symmetric $2 n \times 2 n$ matrices). In the Appendix, we give a brief introduction of this index theory.

Theorem 1.1. Let $H$ satisfy conditions $\left(H_{0}\right)$ and $\left(H_{\infty}\right)$. Suppose $J B_{1}(t)=B_{1}(t) J$ and $B_{0}(t)=H^{\prime \prime}(t, 0)$ satisfying one of the twisted conditions

$$
\begin{aligned}
& B_{1}(t)+k I \leq B_{0}(t), \\
& B_{0}(t)+k I \leq B_{1}(t),
\end{aligned}
$$

for some constant $k \geq \pi$. Then (1-1) possesses at least one nontrivial solution. If $v_{L}\left(B_{0}\right)=0$, the system (1-1) possesses at least two nontrivial solutions.

For the periodic solutions of a asymptotically linear Hamiltonian system, we refer to [Chang 1981; Long 1993; Conley and Zehnder 1984; Liu 2005b]. We note that we only need to prove the case

$$
L=L_{0}=\{0\} \oplus \mathbb{R}^{n}
$$

The reason is that there is an orthogonal symplectic matrix $P$ such that $P L=L_{0}$. All the conditions hold in (1-1) after taking $z(t)=P x(t)$ there. We note that the problem (1-1) is related to the Bolza problem (see [Clarke and Ekeland 1982; Ekeland 1990]).

We should briefly review the general study of the problem (1-1). For a general symplectic manifold $(M, \omega)$ (usually closed, that is, compact without boundary; an example nonclosed case is the cotangent bundle of a closed Riemannian manifold with the zero section as the Lagrangian submanifold) and a closed Lagrangian submanifold $L \subset M$, the problem (1-1) has been widely studied. The multiplicity problems of Hamiltonian systems on a symplectic manifold with Lagrangain boundary values are related to Arnold's conjecture about Lagrangian intersections. The autonomous case of this problem in $\mathbb{R}^{2 n}$ is related to the Arnold chord conjecture. Generally, a Hamiltonian flow starting from a Lagrangian submanifold does not necessary return to the Lagrangian submanifold again. Arnold conjectured that, under some conditions, the Lagrangian intersection number has a lower bound estimated by the sum of all Beti numbers of the Lagrangian submanifold in the nondegenerate case; this sum is in turn estimated by the cup-length of the Lagrangian submanifold (see for example [Conley and Zehnder 1984; Hofer 1988; Floer 1988, 1989; Oh 1995; Ono 1996; Chekanov 1996, 1998; Liu 2005a]). For the Arnold chord conjecture, we mention [Arnold 1986; Mohnke 2001]. The multiplicity of the fixed energy problem (1-1) was studied in [Guo and Liu 2007]. The main differences between this work and the others are that here the symplectic manifold and the Lagrangian submanifold are not compact and all the topological data of the Lagrangian submanifold are trivial. 


\section{Some further properties of the Maslov type index theory}

Liu [2007] developed some important properties of the $L$-index theory. In this section we study the relation between the $L$-index of solutions of Hamiltonian systems with $L$-boundary conditions and the Morse index of the corresponding functional defined via the Galerkin approximation method on the finite-dimensional truncated space at its corresponding critical points. Fei and Qiu [1996] treated the periodic case.

The eigenspace $E_{k}$ of the operator $A=-J d / d t$ in the domain

$$
W_{L_{0}}^{1,2}\left([0,1], \mathbb{R}^{2 n}\right):=\left\{z \in W^{1,2}\left([0,1], \mathbb{R}^{2 n}\right): z(0) \in L_{0}, z(1) \in L_{0}\right\}
$$

can be written as

$$
\begin{aligned}
E_{k} & =-J \exp (k \pi t J) a_{k}=-J\left(\cos (k \pi t) I_{2 n}+J \sin (k \pi t)\right) a_{k}, \\
a_{k} & =\left(a_{k 1}, \cdots, a_{k n}, 0, \cdots, 0\right) \in \mathbb{R}^{2 n} .
\end{aligned}
$$

We define a Hilbert space

$$
\mathrm{W}_{L_{0}}=W_{L_{0}}^{1 / 2,2}\left([0,1], \mathbb{R}^{2 n}\right) \subset \bigoplus_{k \in \mathbb{Z}} E_{k}
$$

with $L_{0}$ boundary conditions

$$
\mathrm{W}_{L_{0}}=\left\{\left.z \in L^{2}\left|z(t)=\sum_{k \in \mathbb{Z}}-J \exp (k \pi t J) a_{k},\|z\|^{2}:=\sum_{k \in \mathbb{Z}}(1+|k|)\right| a_{k}\right|^{2}<\infty\right\} .
$$

We denote its inner product by $\langle\cdot, \cdot\rangle$. By the well-known Sobolev embedding theorem, for any $s \in[1,+\infty)$, there is a constant $C_{s}>0$ such that

$$
\|z\|_{L^{s}} \leq C_{s}\|z\| \quad \text { for all } z \in \mathrm{W}_{L_{0}} .
$$

For any Lagrangian subspace $L \in \Lambda(n)$, suppose $P \in \mathrm{Sp}(2 n) \cap O(2 n)$ such that $L=P L_{0}$. Then we define $\mathrm{W}_{L}=P \mathrm{~W}_{L_{0}}$. We denote by

$$
\mathrm{W}_{L_{0}}^{m}=\bigoplus_{k=-m}^{m} E_{k}=\left\{z \mid z(t)=\sum_{k=-m}^{m}-J \exp (k \pi t J) a_{k}\right\}
$$

the finite dimensional truncation of $\mathrm{W}_{L_{0}}$, and $\mathrm{W}_{L}^{m}=P \mathrm{~W}_{L_{0}}^{m}$.

Let $P^{m}=P_{L}^{m}: \mathrm{W}_{L} \rightarrow \mathrm{W}_{L}^{m}$ be the orthogonal projection for $m \in \mathbb{N}$. Then $\Gamma=\left\{P^{m} ; m \in \mathbb{N}\right\}$ is a Galerkin approximation scheme with respect to $A$ defined in (2-2) below, that is,

$$
P^{m} \rightarrow I \text { strongly as } m \rightarrow \infty \quad \text { and } P^{m} A=A P^{m} .
$$

In this section we still consider the problem (1-1), with $H$ satisfying

$$
\left|H^{\prime \prime}(t, z)\right| \leq a\left(1+|z|^{p}\right) \quad \text { for all }(t, z) \in \mathbb{R} \times \mathbb{R}^{2 n}
$$


and for some $a>0, p>1$. We consider the functional on $\mathrm{W}_{L}$

$$
f(z)=\int_{0}^{1}\left(\frac{1}{2}(-J \dot{z}, z)-H(t, z)\right) d t=\frac{1}{2}\langle A z, z\rangle-g(z), \quad z \in \mathrm{W}_{L} .
$$

A critical point of $f$ on $\mathrm{W}_{L}$ is a solution of (1-1). For a critical point $z=z(t)$, we denote $B(t)=H^{\prime \prime}(t, z(t))$ and define an operator $B$ on $\mathrm{W}_{L}$ by

$$
\langle B z, w\rangle=\int_{0}^{1}(B(t) z, w) d t .
$$

Using the Floquet theory we have

$$
v_{L}(B)=\operatorname{dim} \operatorname{ker}(A-B) .
$$

For $\delta>0$, we denote by $m_{\delta}^{*}(\cdot)$, where $*=+, 0,-$, the dimension of the total eigenspace corresponding to the eigenvalue $\lambda$ belonging to $[\delta,+\infty),(-\delta, \delta)$, $(-\infty,-\delta]$, respectively, and denote by $m^{*}(\cdot)$, where again $*=+, 0,-$ the dimension of the total eigenspace corresponding to the eigenvalue $\lambda$ belonging to $(0,+\infty),\{0\},(-\infty, 0)$, respectively. For any adjoint operator $L$, we define $L^{\sharp}=$ $\left(\left.L\right|_{I m L}\right)^{-1}$, and we also define $P^{m} L P^{m}=\left.\left(P^{m} L P^{m}\right)\right|_{\mathrm{W}_{L}^{m}}$. The following result is adapted from [Fei and Qiu 1996], where the periodic boundary condition was considered (see also [Long 1993]).

Theorem 2.1. For any $B(t) \in C\left([0,1], \mathrm{L}_{s}\left(\mathbb{R}^{2 n}\right)\right)$ having the pair of $L$ indexes $\left(i_{L}(B), v_{L}(B)\right)$ and any constant $0<\delta \leq \frac{1}{4}\left\|(A-B)^{\sharp}\right\|$, there exists $m_{0}>0$ such that for $m \geq m_{0}$, we have

$$
\begin{aligned}
& m_{\delta}^{+}\left(P^{m}(A-B) P^{m}\right)=m n-i_{L}(B)-v_{L}(B), \\
& m_{d}^{-}\left(P^{m}(A-B) P^{m}\right)=m n+i_{L}(B)+n, \\
& m_{\delta}^{0}\left(P^{m}(A-B) P^{m}\right)=v_{L}(B) .
\end{aligned}
$$

Proof. We follow the ideas of [Fei and Qiu 1996].

Step 1. There is an $m_{1}>0$ such that for $m \geq m_{1}$

$$
\operatorname{dim} \operatorname{ker}\left(P^{m}(A-B) P^{m}\right) \leq \operatorname{dim} \operatorname{ker}(A-B) .
$$

In fact, by contradiction it is easy to show that there is a constant $m_{2}>0$ such that for $m \geq m_{2}$

$$
\operatorname{dim} P^{m} \operatorname{ker}(A-B)=\operatorname{dim} \operatorname{ker}(A-B) .
$$

Since $B$ is compact, there is $m_{1} \geq m_{2}$ such that for $m \geq m_{1}$

$$
\left\|\left(I-P^{m}\right) B\right\| \leq 2 \delta .
$$


Take $m \geq m_{1}$, and let $\mathrm{W}_{L}^{m}=P^{m} \operatorname{ker}(A-B) \oplus Y^{m}$. Then $Y^{m} \subset \operatorname{Im}(A-B)$. For $y \in Y^{m}$ we have

$$
y=(A-B)^{\sharp}(A-B) y=(A-B)^{\sharp}\left(P^{m}(A-B) P^{m} y+\left(P^{m}-I\right) B y\right) .
$$

This implies

$$
\|y\| \leq \frac{1}{2 \delta}\left\|P^{m}(A-B) P^{m} y\right\| \quad \text { for all } y \in Y^{m} .
$$

By (2-6) and (2-7) we have (2-5).

Step 2. We distinguish two cases.

Case 1: $v_{L}(B)=0$. By (2-3) and step 1 we obtain for $m \geq m_{1}$ that

$$
m^{0}\left(P^{m}(A-B) P^{m}\right)=\operatorname{dim} \operatorname{ker}(A-B)=0 .
$$

Since $B$ is compact, there exists $m_{3} \geq m_{1}$ such that, for $m \geq m_{3}$,

$$
\left\|\left(I-P^{m}\right) B\right\| \leq \frac{1}{2}\left\|(A-B)^{\sharp}\right\|^{-1} .
$$

Then $P^{m}(A-B) P^{m}=(A-B) P^{m}+\left(I-P^{m}\right) B P^{m}$ implies that

$$
\left\|P^{m}(A-B) P^{m} z\right\| \geq \frac{1}{2}\left\|(A-B)^{\sharp}\right\|^{-1}\|z\| \quad \text { for all } z \in \mathrm{W}_{L}^{m} .
$$

Thus the eigen-subspace $M_{\delta}^{*}\left(P^{m}(A-B) P^{m}\right)$ with eigenvalue $\lambda$ belonging to the intervals $m_{\delta}^{*}\left(P^{m}(A-B) P^{m}\right)$ and the eigen-subspace $M^{*}\left(P^{m}(A-B) P^{m}\right)$ satisfy

$$
M_{\delta}^{*}\left(P^{m}(A-B) P^{m}\right)=M^{*}\left(P^{m}(A-B) P^{m}\right) \text { for } *=+, 0,-.
$$

By Equation (A.5), there is $m_{0} \geq m_{3}$ such that for $m \geq m_{0}$ the relation (2-4) holds. Case 2: $v_{L}(B)>0$. By step 1, it is easy to show that there exists $m_{4}>0$ such that for $m \geq m_{4}$

$$
m_{\delta}^{0}\left(P^{m}(A-B) P^{m}\right) \leq v_{L}(B) .
$$

Let $\gamma \in \mathrm{P}(2 n)$ be the fundamental solution of the linear Hamiltonian system

$$
\dot{z}=J B(t) z \text {. }
$$

Let $\gamma_{s}, 0 \leq s \leq 1$ be the perturbed path defined by Equation (A.4). Define

$$
B_{S}(t)=-J \dot{\gamma}_{S}(t) \gamma_{s}(t)^{-1}, t \in[0,1] .
$$

Let $B_{s}$ be the compact operator defined as $B$ corresponding to $B_{s}(t)$. For $s \neq 0$, there holds $m^{0}\left(A-B_{s}\right)=0$ and $\left\|B_{s}-B\right\| \rightarrow 0$ as $s \rightarrow 0$. If $s \in(0,1]$, we have

$$
i_{L}\left(\gamma_{s}\right)-i_{L}\left(\gamma_{-s}\right)=v_{L}(\gamma)=v_{L}(B), i_{L}\left(\gamma_{-s}\right)=i_{L}(B)=i_{L}(\gamma)
$$


Choose $0<s<1$ such that $\left\|B-B_{ \pm s}\right\| \leq \delta / 2$. By case 1, (2-8), (2-9) and that

$$
P^{m}\left(A-B_{ \pm}\right) P^{m}=P^{m}(A-B) P^{m}+P^{m}\left(B-B_{ \pm}\right) P^{m},
$$

there exists $m_{0} \geq m_{4}$ such that for $m \geq m_{0}$

$$
\begin{aligned}
m_{\delta}^{+}\left(P^{m}(A-B) P^{m}\right) & \leq m^{+}\left(P^{m}\left(A-B_{s}\right) P^{m}\right)=m n-i_{L}(B)-v_{L}(B), \\
m_{\delta}^{+}\left(P^{m}(A-B) P^{m}\right) & \geq m^{+}\left(P^{m}\left(A-B_{-s}\right) P^{m}\right)-m_{\delta}^{0}\left(P^{m}(A-B) P^{m}\right) \\
& \geq m n-i_{L}(B)-v_{L}(B) .
\end{aligned}
$$

Hence, $m_{\delta}^{0}\left(P^{m}(A-B) P^{m}\right)=v_{L}(B)$ and

$$
m_{\delta}^{+}\left(P^{m}(A-B) P^{m}\right)=m n-i_{L}(B)-v_{L}(B) .
$$

Note that $\operatorname{dim} \mathrm{W}_{L}^{m}=(2 m+1) n$, so

$$
m_{\delta}^{-}\left(P^{m}(A-B) P^{m}\right)=m n+n+i_{L}(B) .
$$

Corollary 2.2. Let $B_{j}(t) \in C\left([0,1], \mathrm{L}_{s}\left(\mathbb{R}^{2 n}\right)\right), j=1,2$. Assume $B_{1}(t)<B_{2}(t)$, that is, $B_{2}(t)-B_{1}(t)$ is positive definite for all $t \in[0,1]$. Then there holds

$$
i_{L}\left(B_{1}\right)+v_{L}\left(B_{1}\right) \leq i_{L}\left(B_{2}\right) .
$$

Proof. Just as in Theorem 2.1, corresponding to $B_{j}(t)$ we have the operator $B_{j}$. Let $\Gamma=\left\{P^{m}\right\}$ be the approximation scheme with respect to the operator $A$. Then by (2-4), there exists $m_{0}>0$ such that if $m \geq m_{0}$ there holds

$$
\begin{aligned}
& m_{\delta}^{-}\left(P^{m}\left(A-B_{1}\right) P^{m}\right)=m n+n+i_{L}\left(B_{1}\right), \\
& m_{\delta}^{-}\left(P^{m}\left(A-B_{2}\right) P^{m}\right)=m n+n+i_{L}\left(B_{2}\right),
\end{aligned}
$$

where we choose $0<\delta<\left\|B_{2}-B_{1}\right\| / 2$. Since $A-B_{2}=\left(A-B_{1}\right)-\left(B_{2}-B_{1}\right)$ and $B_{2}-B_{1}$ is positive definite in $\mathrm{W}_{L}^{m}=P^{m} \mathrm{~W}_{L}$ and $\left\langle\left(B_{2}-B_{1}\right) x, x\right\rangle \geq 2 \delta\|x\|$, we have $\left\langle\left(P^{m}\left(A-B_{2}\right) P^{m}\right) x, x\right\rangle \leq-\delta\|x\|$ with

$$
x \in M_{\delta}^{-}\left(P^{m}\left(A-B_{1}\right) P^{m}\right) \oplus M_{\delta}^{0}\left(P^{m}\left(A-B_{1}\right) P^{m}\right) .
$$

This implies that $m n+n+i_{L}\left(B_{1}\right)+v_{L}\left(B_{1}\right) \leq m n+n+i_{L}\left(B_{2}\right)$.

Remark. From the proof of Corollary 2.2, it is easy to show that if $B_{1}(t) \leq B_{2}(t)$ for all $0 \leq t \leq 1$,

$$
i_{L}\left(B_{1}\right) \leq i_{L}\left(B_{2}\right), \quad i_{L}\left(B_{1}\right)+v_{L}\left(B_{1}\right) \leq i_{L}\left(B_{2}\right)+v_{L}\left(B_{2}\right) .
$$

Definition 2.3. For any two matrix functions $B_{j} \in C\left([0,1], \mathrm{L}_{s}\left(\mathbb{R}^{2 n}\right)\right), j=0,1$ with $B_{0}(t)<B_{1}(t)$ for all $t \in \mathbb{R}$, we define

$$
I_{L}\left(B_{0}, B_{1}\right)=\sum_{s \in[0,1)} v_{L}\left((1-s) B_{0}+s B_{1}\right) .
$$


Theorem 2.4. For any two matrix functions $B_{j} \in C\left([0,1], \mathrm{L}_{s}\left(\mathbb{R}^{2 n}\right)\right)$ with $B_{0}(t)<$ $B_{1}(t)$ for all $t \in \mathbb{R}$, we have

$$
I_{L}\left(B_{0}, B_{1}\right)=i_{L}\left(B_{1}\right)-i_{L}\left(B_{0}\right) .
$$

So we call $I_{L}\left(B_{0}, B_{1}\right)$ the relative $L$-index of the pair $\left(B_{0}, B_{1}\right)$.

Proof. Step 1. By Corollary 2.2, if we denote $i_{L}(\lambda)=i_{L}\left((1-\lambda) B_{0}+\lambda B_{1}\right)$, $v_{L}(\lambda)=v_{L}\left((1-\lambda) B_{0}+\lambda B_{1}\right)$, there holds

$$
i_{L}\left(\lambda_{2}\right) \geq i_{L}\left(\lambda_{1}\right)+v_{L}\left(\lambda_{1}\right), \text { for } \lambda_{2}>\lambda_{1} .
$$

So the function $i_{L}(\lambda)$ is a monotone function in $[0,1]$.

Step 2. We prove that for any $\lambda \in[0,1)$ there holds

$$
i_{L}(\lambda+0)=i_{L}(\lambda)+v_{L}(\lambda)
$$

where $i_{L}(\lambda+0)$ is the right limit of $i_{L}(s)$ at $\lambda$. In fact, by (2-11), we have $i_{L}(\lambda)+$ $v_{L}(\lambda) \leq i_{L}(\lambda+0)$. We now use the saddle point reduction methods to prove the opposite inequality $i_{L}(\lambda)+v_{L}(\lambda) \geq i_{L}(\lambda+0)$. Define $B_{\lambda}(t)=(1-\lambda) B_{0}(t)+\lambda B_{1}(t)$. We define in $L^{2}\left([0,1], \mathbb{R}^{2 n}\right)$

$$
f_{\lambda}(x)=\int_{0}^{1}\left[(-J \dot{x}(t), x(t))-\left(B_{\lambda}(t) x(t), x(t)\right)\right] d t \quad \text { for all } x \in \operatorname{dom}(A)=W_{L} .
$$

Then by the saddle point reduction methods (see Equation (A.5)), we can reduce the functional $f_{\lambda}$ in $L^{2}\left([0,1], \mathbb{R}^{2 n}\right)$ to a finite-dimensional subspace $X$ of $L^{2}\left([0,1], \mathbb{R}^{2 n}\right)$ by $a_{\lambda}(x)=f_{\lambda}\left(u_{\lambda}(x)\right)$, where $u_{\lambda}: X \rightarrow L^{2}\left([0,1], \mathbb{R}^{2 n}\right)$ is injective, and $a_{\lambda}$ is continuous in $\lambda$. Denote the Morse indices of $a_{\lambda}$ on $X$ at $x=0$ by $m_{\lambda}^{-}$, $m_{\lambda}^{0}$ and $m_{\lambda}^{-}$. If $\operatorname{dim} X=2 d+n$ large enough, we have from (A.5)

$$
m_{\lambda}^{-}=d+n+i_{L}(\lambda), \quad m_{\lambda}^{0}=v_{L}(\lambda), \quad m_{\lambda}^{+}=d-i_{L}(\lambda)-v_{L}(\lambda) .
$$

For any fixed $\lambda \in[0,1)$, choosing $\mu \in(\lambda, 1) \cup[0, \lambda)$ sufficiently close to $\lambda$, we obtain

$$
m_{\lambda}^{ \pm} \leq m_{\mu}^{ \pm} \leq m_{\lambda}^{ \pm}+v_{L}(\lambda)
$$

Then by (2-12), we have $i_{L}(\lambda) \leq i_{L}(\mu)$ and $i_{L}(\lambda)+v_{L}(\lambda) \geq i_{L}(\mu)$. This implies $i_{L}(\lambda)+v_{L}(\lambda) \geq i_{L}(\lambda+0)$ and $i_{L}(\lambda) \leq i_{L}(\lambda-0)$. But by (2-11), we have $i_{L}(\lambda) \geq i_{L}(\lambda-0)$, so $i_{L}(\lambda)=i_{L}(\lambda-0)$. That is to say, the function $i_{L}(\lambda)$ is left continuous at $(0,1]$. Moreover if $m_{\lambda}^{0}=m^{0}$ is constant in some interval $\left[\lambda_{1}, \lambda_{2}\right]$, then $m_{\lambda}^{-}=m^{-}$and $m_{\lambda}^{+}=m^{+}$are constant in this interval. Thus the function $i_{L}(\lambda)$ is locally constant at its continuous points, its discontinuous points are those with with $v_{L}(\lambda)>0$, and there holds

$$
i_{L}(1)=i_{L}(0)+\sum_{0 \leq \lambda<1} v_{L}(\lambda)
$$


which is exactly $(2-10)$.

Corollary 2.5. If $\gamma \in \mathrm{P}(2 n)$ is the fundamental solution of the linear Hamiltonian system with respect to $B(t)>0$, there holds

$$
i_{L}(\gamma)=\sum_{0<t<1} \operatorname{dim}(\gamma(t) L \cap L) .
$$

Thus we can understand the index $i_{L}(\gamma)$ as a kind of intersection number of the two Lagrangian paths $w(t)=\gamma(t) L$ and $w_{0}(t)=L$.

Proof. We take $B_{1}(t)=B(t)$ and $B_{0}(t)=0$ in Theorem 2.4. We note that the fundamental solution corresponding to $B_{0}(t)=0$ is the constant path $I$. We have

$$
I_{L}(0, B)=i_{L}(\gamma)-i_{L}(I) .
$$

But $i_{L}(I)=i_{L_{0}}(I)=-n$ and $B_{s}(t)=(1-s) B_{0}(t)+s B_{1}(t)=s B(t)$. The corresponding fundamental solution corresponding to $B_{s}(t)=s B(t)$ is $\gamma(s t)$. Thus

$$
I_{L}(0, B)=\sum_{s \in[0,1)} v_{L}(s B)=\sum_{s \in[0,1)} \operatorname{dim}[(\gamma(s) L) \cap L] .
$$

But $\operatorname{dim}[(\gamma(0) L) \cap L]=\operatorname{dim} L=n$, so we have $(2-13)$.

\section{Dual index theory for linear Hamiltonian systems}

Let $B \in C\left([0,1], \mathrm{L}_{s}\left(\mathbb{R}^{2 n}\right)\right)$. Recall that $\mathrm{L}_{s}\left(\mathbb{R}^{2 n}\right)$ is the set of symmetric $2 n \times 2 n$ metrics. Consider the linear Hamiltonian system

$$
\dot{z}=J B(t) z, \quad z \in \mathbb{R}^{2 n} .
$$

We consider in this section the dual Morse index theory of system (3-1) with Lagrangian boundary condition. The dual Morse index theory for periodic boundary condition was studied by Girardi and Matzeu [1991] for the cases of superquadatic Hamiltonian systems, and by the author in [Liu 2001] for the subquadratic Hamiltonian systems. This theory is an application of the Morse-Ekeland index theory [Ekeland 1990]. The dual action principal in Hamiltonian framework was first established by Clarke [1978; 1979; 1981] and Clarke and Ekeland [1978; 1980], and has since been adapted by many mathematicians to the study of various variational problems. The index theory for convex Hamiltonian systems was established by I. Ekeland (see for example [1990]), whose works are of fundamental importance in the study of convex Hamiltonian systems.

Let $W_{L}$ be the Hilbert space defined by

$$
W_{L}=\left\{z=(x, y)^{T} \in W^{1,2}\left([0,1], \mathbb{R}^{2 n}\right) \mid z(0), z(1) \in L\right\} \subset L^{2} .
$$


The embedding $j: W_{L} \rightarrow \mathrm{L}=L^{2}\left([0,1], \mathbb{R}^{2 n}\right)$ is compact. Denote by $\langle\cdot, \cdot\rangle$ and $\langle\cdot, \cdot\rangle_{2}$ the respective inner products on $W_{L}$ and $\mathrm{L}$. We define an operator $A: \mathrm{L} \rightarrow \mathrm{L}$ with domain $W_{L}$ by $A=-J d / d t$. The spectrum of $A$ is isolated, and in fact, $\sigma(A)=\pi \mathbb{Z}$. Let $k \notin \sigma(A)$ be so large such that $B(t)+k I>0$. Then the operator $\Lambda_{k}=A+k I: W_{L} \rightarrow \mathrm{L}$ is invertible, and its inverse is compact. We define a quadratic form in $\mathrm{L}$ by

$$
Q_{k, B}^{*}(v, u)=\int_{0}^{1}\left(\left(C_{k}(t) v(t), u(t)\right)-\left(\Lambda_{k}^{-1} v(t), u(t)\right)\right) d t \quad \text { for all } v, u \in \mathrm{L},
$$

where $C_{k}(t)=(B(t)+k I)^{-1}$. Define $Q_{k, B}^{*}(v)=Q_{k, B}^{*}(v, v)$. Then

$$
\left\langle C_{k} v, v\right\rangle_{2}=\int_{0}^{1}\left(C_{k}(t) v(t), v(t)\right) d t
$$

defines a Hilbert structure in L. $C_{k}^{-1} \Lambda_{k}^{-1}$ is a self-adjoint and compact operator under this inner product. By the spectral theory, there exists a basis $e_{j}, j \in \mathbb{N}$ of $\mathrm{L}$, and an eigenvalue sequence $\lambda_{j} \rightarrow 0$ in $\mathbb{R}$ such that

$$
\begin{aligned}
\left\langle C_{k} e_{i}, e_{j}\right\rangle_{2} & =\delta_{i j}, \\
\left\langle\Lambda_{k}^{-1} e_{j}, v\right\rangle_{2} & =\left\langle C_{k} \lambda_{j} e_{j}, v\right\rangle_{2} \quad \text { for all } v \in \mathrm{L} .
\end{aligned}
$$

For any $v \in \mathrm{L}$ with $v=\sum_{j=1}^{\infty} \xi_{j} e_{j}$, there holds

$$
Q_{k, B}^{*}(v)=-\int_{0}^{1}\left(\Lambda_{k}^{-1} v(t), v(t)\right)-\left(C_{k}(t) v(t), v(t)\right) d t=\sum_{j=1}^{\infty}\left(1-\lambda_{j}\right) \xi_{j}^{2} .
$$

Define

$$
\begin{aligned}
& \mathrm{L}_{k}^{-}(B)=\left\{\sum_{j=1}^{\infty} \xi_{j} e_{j} \mid \xi_{j}=0 \text { if } 1-\lambda_{j} \geq 0\right\}, \\
& \mathrm{L}_{k}^{0}(B)=\left\{\sum_{j=1}^{\infty} \xi_{j} e_{j} \mid \xi_{j}=0 \text { if } 1-\lambda_{j} \neq 0\right\}, \\
& \mathrm{L}_{k}^{+}(B)=\left\{\sum_{j=1}^{\infty} \xi_{j} e_{j} \mid \xi_{j}=0 \text { if } 1-\lambda_{j} \leq 0\right\} .
\end{aligned}
$$

Observe that $\mathrm{L}_{k}^{-}(B), \mathrm{L}_{k}^{0}(B)$ and $\mathrm{L}_{k}^{+}(B)$ are $Q_{k, B}^{*}$-orthogonal, and also that $\mathrm{L}=$ $\mathrm{L}_{k}^{-}(B) \oplus \mathrm{L}_{k}^{0}(B) \oplus \mathrm{L}_{k}^{+}(B)$. Since $\lambda_{j} \rightarrow 0$ as $j \rightarrow \infty$, both $\mathrm{L}_{k}^{-}(B)$ and $\mathrm{L}_{k}^{0}(B)$ are finite subspaces. We define the $k$-dual Morse index of $B$ by

$$
i_{k}^{*}(B)=\operatorname{dim} \mathrm{L}_{k}^{-}(B), \quad v_{k}^{*}(B)=\operatorname{dim} \mathrm{L}_{k}^{0}(B) .
$$

Theorem 3.1. There holds

$$
i_{k}^{*}(B)=i_{L}(B)+n+n\left[\frac{k}{\pi}\right], \quad v_{k}^{*}(B)=v_{L}(B),
$$


where $[a]=\max \{j \in \mathbb{Z} \mid j \leq a\}$.

Proof. We only prove (3-2) for the special case $L=L_{0}$. We first define a functional on

$$
W^{m}=\left\{x \mid x(t)=\sum_{j=-m}^{m}-J \exp (j \pi t J) a_{j}, a_{j} \in \mathbb{R}^{n} \oplus\{0\} \subset \mathbb{R}^{2 n}\right\}
$$

by

$$
\begin{aligned}
Q_{m}(x) & =\int_{0}^{1}\left[\left(\Lambda_{k} x(t), x(t)\right)-\left(C_{k}^{-1}(t) x, x\right)\right] d t \\
& =\int_{0}^{1}[(-J \dot{x}(t), x(t))-(B(t) x(t), x(t))] d t \quad \text { for all } x \in W^{m} .
\end{aligned}
$$

We define two linear operators $A_{k}$ and $B_{k}$ from $W^{m}$ onto its dual space $W^{m *} \cong W^{m}$ such that

$$
\begin{array}{ll}
\left\langle A_{k} x, y\right\rangle_{2}=\int_{0}^{1}\left(\Lambda_{k} x(t), y(t)\right) d t & \text { for all } x, y \in W^{m}, \\
\left\langle B_{k} x, y\right\rangle_{2}=\int_{0}^{1}((B(t)+k I) x(t), y(t)) d t & \text { for all } x, y \in W^{m} .
\end{array}
$$

Next $\langle\cdot, \cdot\rangle_{m}:=\left\langle B_{k} \cdot, \cdot\right\rangle_{2}$ is a inner product in $W^{m}$. We consider the eigenvalues $\mu_{j} \in \mathbb{R}$ of $A_{k}$ with respect to this inner product, that is,

$$
A_{k} x_{j}=\mu_{j} B_{k} x_{j}
$$

for some $x_{j} \in W^{m} \backslash\{0\}$. Suppose $\mu_{1} \leq \mu_{2} \leq \cdots \leq \mu_{l}$ with $l=\operatorname{dim} W^{m}=2 m n+n$ (each eigenvalue is counted with its multiplicity), and construct a basis in $W^{m}$ of eigenvectors $v_{1}, \ldots, v_{l}$ such that, for $i, j=1,2, \ldots, l$,

$$
\begin{aligned}
\left\langle v_{i}, v_{j}\right\rangle_{m} & =\delta_{i j}, \\
\left\langle A_{m} v_{i}, v_{j}\right\rangle_{m} & =\mu_{i} \delta_{i j}, \\
Q_{m}\left(v_{i}, v_{j}\right) & =\left(\mu_{i}-1\right) \delta_{i j} .
\end{aligned}
$$

The Morse indexes $m^{-}\left(Q_{m}\right), m^{0}\left(Q_{m}\right)$ and $m^{+}\left(Q_{m}\right)$ of $Q_{m}$ satisfy

$$
\begin{aligned}
& m^{-}\left(Q_{m}\right)={ }^{\sharp}\left\{\mu_{j} \mid 1 \leq j \leq l, \mu_{j}<1\right\}, \\
& m^{+}\left(Q_{m}\right)={ }^{\sharp}\left\{\mu_{j} \mid 1 \leq j \leq l, \mu_{j}>1\right\}, \\
& m^{0}\left(Q_{m}\right)={ }^{\sharp}\left\{\mu_{j} \mid 1 \leq j \leq l, \mu_{j}=1\right\} .
\end{aligned}
$$

By Theorem 2.1, we have for $m>0$ large enough

$$
m^{-}\left(Q_{m}\right)=m n+n+i_{L}(B), \quad m^{0}\left(Q_{m}\right)=v_{L}(B) .
$$


We denote by $Q_{k, m}^{*}$ the restriction of the quadratic $Q_{k}^{*}$ to the subspace $W^{m}$, and define $i_{k, m}^{*}(B)=m^{-}\left(Q_{k, m}^{*}\right), v_{k, m}^{*}(B)=m^{0}\left(Q_{k, m}^{*}\right)$. By an argument from [Girardi and Matzeu 1991], we have $i_{k, m}^{*}(B) \rightarrow i_{k}^{*}(B)$ and $v_{k, m}^{*}(B) \rightarrow v_{k}^{*}(B)$ as $m \rightarrow \infty$. Let $v_{j}^{\prime}=A_{m} v_{j}$ for $j=1,2, \ldots, l$. It is a basis of $W^{m}$ and

$$
Q_{k, m}^{*}\left(v_{i}^{\prime}, v_{j}^{\prime}\right)= \begin{cases}0, & \text { for } i \neq j, \\ \mu_{j}\left(\mu_{j}-1\right), & \text { for } i=j .\end{cases}
$$

$Q_{k, m}^{*}\left(v_{j}^{\prime}\right)$ is negative if and only if $0<\mu_{j}<1$. We now deduce the total multiplicity of the negative eigenvalues $\mu_{j}<0$. If one replaces the inner product $\langle\cdot, \cdot\rangle_{m}$ by the usual one, that is , one replaces the matrix $B_{k}$ by the identity $I$, the eigenvalues $\mu_{j}$ should be replaced by the eigenvalues $\eta_{j}$ of $A_{m}$ with respect to the standard inner product. It is easy to check that $\mu_{j}$ and $\eta_{j}$ have the same signs. So the total multiplicity of negative $\mu_{j}$ 's equals the total multiplicity of negative $\eta_{h}$ 's. But we have

$$
\eta_{h}=h \pi+k, \quad-m \leq h \leq m,
$$

and each has multiplicity $n$. Therefore, the total multiplicity of the negative $\eta_{h}$ is $n(m-[k / \pi])$. So the total multiplicity of $\mu_{j} \in(0,1)$ is $m^{-}\left(Q_{m}\right)-n(m-[k / \pi])$. By definition we have

$$
i_{k, m}^{*}(B)=m^{-}\left(Q_{m}\right)-n(m-[k / \pi]) .
$$

So for $m>0$ large enough, from (3-3) we get (3-2).

Corollary 3.2. 3.2 Under the condition of Equation (2-3), there holds

$$
I_{L}\left(B_{0}, B_{1}\right)=i_{k}^{*}\left(B_{1}\right)-i_{k}^{*}\left(B_{0}\right) .
$$

\section{Proof of Theorem 1.1 and some consequences}

Lemma 4.1 [Chang 1981, Theorem 5.1, Corollary II.5.2]. Let $f \in C^{2}(\mathrm{~L}, \mathbb{R})$ satisfy the $(P S)$ condition $f^{\prime}(0)=0$ and suppose there exists

$$
r \notin\left[m^{-}\left(f^{\prime \prime}(0)\right), m^{-}\left(f^{\prime \prime}(0)\right)+m^{0}\left(f^{\prime \prime}(0)\right)\right]
$$

with $H_{q}\left(\mathrm{~L}, f_{a} ; \mathbb{R}\right) \cong \delta_{q, r} \mathbb{R}$. Then $f$ has at least one nontrivial critical point $u_{1} \neq 0$. Moreover, if $m^{0}\left(f^{\prime \prime}(0)\right)=0$ and $m^{0}\left(f^{\prime \prime}\left(u_{1}\right)\right) \leq\left|r-m^{-}\left(f^{\prime \prime}(0)\right)\right|$, then $f$ has one more nontrivial critical point $u_{2} \neq u_{1}$.

Theorem 1.1. Without loss any generality we can suppose $H(t, 0)=0$ and $L=L_{0}$. By the condition $\left(H_{\infty}\right)$ and the remark after Equation $(2-1)$, we get that $i_{L}\left(B_{1}\right)+$ $v_{L}\left(B_{1}\right) \leq i_{L}\left(B_{2}\right)+v_{L}\left(B_{2}\right)$, and so we have $v_{L}\left(B_{1}\right)=0$. We shall first prove that under the above conditions (1-2) or (1-3), there holds

$$
i_{L}\left(B_{1}\right) \notin\left[i_{L}\left(B_{0}\right), i_{L}\left(B_{0}\right)+v_{L}\left(B_{0}\right)\right] .
$$


More clearly, under the condition (1-2), it is claimed

$$
i_{L}\left(B_{1}\right)=i_{L}\left(B_{1}\right)+v_{L}\left(B_{1}\right)<i_{L}\left(B_{0}\right),
$$

and under the condition (1-3), it is claimed

$$
i_{L}\left(B_{0}\right)+v_{L}\left(B_{0}\right)<i_{L}\left(B_{1}\right) .
$$

We first prove (4-1). By Equation (2-1) and condition (1-2), we have

$$
i_{L}\left(B_{1}\right) \leq i_{L}\left(B_{1}+k I\right) \leq i_{L}\left(B_{0}\right) .
$$

We shall prove

$$
i_{L}\left(B_{1}\right)<i_{L}\left(B_{1}+k I\right) .
$$

In fact, suppose

$$
\gamma_{1}(t)=\left(\begin{array}{ll}
S_{1}(t) & V_{1}(t) \\
T_{1}(t) & U_{1}(t)
\end{array}\right) \in \mathrm{P}(2 n)
$$

is a symplectic path that is the fundamental solution of the linear Hamiltonian system associated with the matrix function $B_{1}(t)$. Since $J B_{1}(t)=B_{1}(t) J$, one can show that $\exp (J k t) \gamma_{1}(t)$ is the fundamental solution of the linear Hamiltonian system

$$
\dot{z}=J\left(B_{1}(t)+k I\right) z
$$

One has

$$
\exp (J k t) \gamma_{1}(t)=\left(\begin{array}{ll}
S_{1}(t) \cos k t-T_{1}(t) \sin k t & V_{1}(t) \cos k t-U_{1}(t) \sin k t \\
S_{1}(t) \sin k t+T_{1}(t) \cos k t & V_{1}(t) \sin k t+U_{1}(t) \cos k t
\end{array}\right) .
$$

The associated unitary $n \times n$ matrix $\mathrm{Q}(t)$ defined by (2-2) with respect to the above matrix is

$$
\begin{aligned}
\mathrm{Q}(t) & =\left[U_{1}(t)-\sqrt{-1} V_{1}(t)\right]\left[U_{1}(t)+\sqrt{-1} V_{1}(t)\right]^{-1} \exp (2 k \sqrt{-1} t) \\
& =\mathrm{Q}_{1}(t) \exp (2 k \sqrt{-1} t) .
\end{aligned}
$$

In Equation (A.6), $\Delta_{j}=\theta_{j}(1)-\theta_{j}(0)$ and $\Delta_{j}^{1}=\theta_{j}^{1}(1)-\theta_{j}^{1}(0)$, associated respectively to $\mathrm{Q}(t)$ and $\mathrm{Q}_{1}(t)$, satisfy

$$
\Delta_{j}=\theta_{j}(1)-\theta_{j}(0)=\Delta_{j}^{1}+2 k=\theta_{j}^{1}(1)-\theta_{j}^{1}(0)+2 k .
$$

Since $k \geq \pi$, there holds

$$
i_{L}\left(B_{1}\right)+n \leq i_{L}\left(B_{1}+k I\right) .
$$

Thus we have proved (4-1), and (4-2) can be proved similarly.

By the condition $\left(H_{\infty}\right), H^{\prime \prime}(t, x)$ is bounded and there exist $\mu_{1}, \mu>0$ such that

$$
I \leq H^{\prime \prime}(t, x)+\mu I \leq \mu_{1} I \quad \text { for all }(t, x) .
$$


We define a convex function $N(t, x)=H(t, x)+\mu|x|^{2} / 2$. Its Fenchel dual defined by

$$
N^{*}(t, x)=\sup _{y \in \mathbb{R}^{2 n}}\{(x, y)-N(t, y)\}
$$

satisfies (see [Ekeland 1990])

$$
\begin{gathered}
N^{*} \in C^{2}\left([0,1] \times \mathbb{R}^{2 n}, \mathbb{R}\right), \\
N^{* \prime \prime}(t, y)=N^{\prime \prime}(t, x)^{-1} \quad \text { for } y=N^{\prime}(t, x) .
\end{gathered}
$$

From (4-4) we have

$$
\mu_{1}^{-1} I \leq N^{* \prime \prime}(t, y) \leq I \text { for all }(t, y) .
$$

So we have $|x| \rightarrow \infty$ if and only if $|y| \rightarrow \infty$ with $y=N^{\prime}(t, x)$. Thus there exists $r_{1}>0$ such that

$$
\left(B_{2}(t)+\mu I\right)^{-1} \leq N^{* \prime \prime}(t, y) \leq\left(B_{1}(t)+\mu I\right)^{-1}
$$

for all $t, y$ with $|y| \geq r_{1}$. We choose $\mu>0$ satisfying (4-4) and $\mu \notin \sigma(A)$. We recall that $\left(\Lambda_{\mu} x\right)(t)=-J \dot{x}(t)+\mu x(t)$. We consider the functional

$$
f(u)=-\frac{1}{2} \int_{0}^{1}\left[\left(\Lambda_{\mu}^{-1} u(t), u(t)\right)-N^{*}(t, u(t))\right] d t \quad \text { for } u \in \mathrm{L} .
$$

It is easy to see that $f \in C^{2}$ and satisfies (PS) condition (see [Ekeland 1990]). There is a one to one correspondence from the critical points of $f$ to the solutions of Hamiltonian systems (1-1). We note that 0 is a trivial critical point of $f$ and $N^{* \prime}(t, 0)=0$. At every critical point $u_{0}$, the second variation of $f$ defines a quadratic form on L by

$\left(f^{\prime \prime}\left(u_{0}\right) u, u\right)=-\int_{0}^{1}\left[\left(\Lambda_{\mu}^{-1} u(t), u(t)\right)-\left(N^{* \prime \prime}\left(t, u_{0}(t)\right) u(t), u(t)\right)\right] d t \quad$ for $u \in \mathrm{L}$.

Its Morse index and nullity are both finite we denote by $\left(i_{\mu}^{*}\left(u_{0}\right), v_{\mu}^{*}\left(u_{0}\right)\right)$ the index pair. The critical point $u_{0}$ corresponds to a solution $x_{0}=\Lambda_{\mu}^{-1} u_{0}$ of (1-1), and $N^{* \prime \prime}\left(t, u_{0}(t)\right)=N^{\prime \prime}\left(t, x_{0}(t)\right)^{-1}$. So by Theorem 3.1, we have

$$
i_{\mu}^{*}\left(u_{0}\right)=i_{L}\left(x_{0}\right)+n+n\left[\frac{\mu}{\pi}\right], v_{\mu}^{*}\left(u_{0}\right)=v_{L}\left(x_{0}\right) .
$$

The index pair $\left(i_{L}\left(x_{0}\right), v_{L}\left(x_{0}\right)\right)$ is the $L$-index of the linear Hamiltonian system

$$
\dot{y}(t)=J H^{\prime \prime}\left(t, x_{0}(t)\right) y(t) .
$$

By condition (1-2) and the result (4-3), we have

$$
i_{L}\left(B_{1}\right)+v_{L}\left(B_{1}\right)+n \leq i_{L}\left(B_{0}\right) .
$$


By condition (1-3), similarly we have

$$
i_{L}\left(B_{0}\right)+v_{L}\left(B_{0}\right)+n \leq i_{L}\left(B_{1}\right) .
$$

From (4-7) and the above inequality, we have that

$$
\left|i_{L}\left(B_{0}\right)-i_{L}\left(B_{1}\right)\right| \geq n \quad \text { and } \quad\left|i_{\mu}^{*}\left(B_{0}\right)-i_{\mu}^{*}\left(B_{1}\right)\right| \geq n .
$$

In the following, we need to prove that the homology groups satisfy

$$
H_{q}\left(\mathrm{~L}, f_{a} ; \mathbb{R}\right) \cong \delta_{q r} \mathbb{R}, \quad q=0,1, \ldots,
$$

for some $a \in \mathbb{R}$ and $r=i_{\mu}^{*}\left(B_{1}\right) . f_{a}=\{x \in \mathrm{L} \mid f(x) \leq a\}$ is the level set below $a$. We follow the ideas of the proof of Lemma II.5.1 in [Chang 1981] to prove (4-9). See [Dong 2005] and [Liu 2005b] for some similar computations.

Step 1. Under the condition $\left(H_{\infty}\right)$, there holds

$$
\mathrm{L}=\mathrm{L}_{\mu}^{-}\left(B_{1}\right) \oplus \mathrm{L}_{\mu}^{+}\left(B_{2}\right),
$$

where $\mathrm{L}_{\mu}^{*}(B)$ for $*= \pm, 0$ is defined in Section 3. In fact, it is clear that $\mathrm{L}_{\mu}^{-}\left(B_{1}\right) \cap$ $\mathrm{L}_{\mu}^{+}\left(B_{2}\right)=\{0\}$. By $v_{\mu}^{*}\left(B_{2}\right)=v_{L}\left(B_{2}\right)=0$, we have $\mathrm{L}=\mathrm{L}_{\mu}^{-}\left(B_{2}\right) \oplus \mathrm{L}_{\mu}^{+}\left(B_{2}\right)$. By condition $\left(H_{\infty}\right)$, we have $i_{\mu}^{*}\left(B_{1}\right)=i_{\mu}^{*}\left(B_{2}\right)=r$. Suppose $\xi_{1}, \xi_{2}, \cdots, \xi_{r}$ is a basis in $\mathrm{L}_{\mu}^{-}\left(B_{1}\right)$. Decompose $\xi_{j}$ by $\xi_{j}=\xi_{j}^{-}+\xi_{j}^{+}$with $\xi_{j} \in \mathrm{L}_{\mu}^{ \pm}\left(B_{2}\right)$. It is clear that $\xi_{1}^{-}, \cdots, \xi_{r}^{-}$are linear independent, so it is a basis for $\mathrm{L}_{\mu}^{-}\left(B_{2}\right)$. For any $\xi \in \mathrm{L}$, there holds $\xi=\xi^{-}+\xi^{+}$with $\xi^{ \pm} \in \mathrm{L}_{\mu}^{ \pm}\left(B_{2}\right)$. Suppose $\xi^{-}=a_{1} \xi_{1}^{-}+\cdots+a_{r} \xi_{r}^{-}$. Then

$$
\xi=\sum_{j=1}^{r} a_{j} \xi_{j}+\left(\xi^{+}-\sum_{j=1}^{r} a_{j} \xi_{j}^{+}\right)=\xi_{1}+\xi_{2}
$$

with $\xi_{1} \in \mathrm{L}_{\mu}^{-}\left(B_{1}\right)$ and $\xi_{2} \in \mathrm{L}_{\mu}^{+}\left(B_{2}\right)$.

Step 2. For sufficiently small $s>0$, from the structure of the symplectic group and the definition of the Maslov-type index, we know that $v_{L}\left(B_{1}-s I\right)=v_{L}\left(B_{1}\right)=0$, and $v_{L}\left(B_{2}+s I\right)=v_{L}\left(B_{2}\right)=0$, and so $i_{L}\left(B_{1}-s I\right)=i_{L}\left(B_{1}\right)=i_{L}\left(B_{2}\right)=i_{L}\left(B_{2}+s I\right)$. Denote the so-called deformation space by

$$
D_{R}=\mathrm{L}_{\mu}^{-}\left(B_{1}-s I\right) \oplus\left\{u \in \mathrm{L}_{\mu}^{+}\left(B_{2}+s I\right) \mid\|u\| \leq R\right\} .
$$

For $R>0$ and $-a>0$ large, we have the deformation result

$$
H_{q}\left(\mathrm{~L}, f_{a} ; \mathbb{R}\right)=H_{q}\left(D_{R}, D_{R} \cap f_{a} ; \mathbb{R}\right) .
$$

The proof of (4-10) is standard in the Morse theory [Bott 1982]. We only need to use the negative flow to deform $\left(\mathrm{L}, f_{a}\right)$ to $\left(D_{R}, D_{R} \cap f_{a}\right)$. For any $u=u_{1}+u_{2} \in \mathrm{L}$ 
with $u_{1} \in \mathrm{L}_{\mu}^{-}\left(B_{1}-s I\right)$ and $u_{2} \in \mathrm{L}_{\mu}^{+}\left(B_{2}+s I\right)$, by the self-adjointness, we have

$$
\begin{aligned}
\left(f^{\prime}(u), u_{2}-u_{1}\right)= & -\int_{0}^{1} d t\left[\left(\Lambda^{-1} u, u_{2}-u_{1}\right)-\left(N^{* \prime}(t, u), u_{2}-u_{1}\right)\right] \\
= & \int_{0}^{1} d t\left[\left(\Lambda^{-1} u_{1}, u_{1}\right)-\left(\Lambda^{-1} u_{2}, u_{2}\right)\right] \\
& \quad+\int_{0}^{1} d t\left(\int_{0}^{1} d \tau N^{* \prime \prime}(t, \tau u)\left(u_{1}+u_{2}\right), u_{2}-u_{1}\right) \\
= & \int_{0}^{1} d t\left(\Lambda^{-1} u_{1}, u_{1}\right)-\int_{0}^{1} d t\left(\int_{0}^{1} d \tau N^{* \prime \prime}(t, \tau u) u_{1}, u_{1}\right) \\
& \quad-\int_{0}^{1} d t\left(\Lambda^{-1} u_{2}, u_{2}\right)+\int_{0}^{1} d t\left(\int_{0}^{1} d \tau N^{* \prime \prime}(t, \tau u) u_{2}, u_{2}\right) .
\end{aligned}
$$

By (4-5) and (4-6), we have

$$
\begin{array}{rl}
\int_{0}^{1} & d t\left(\int_{0}^{1} d \tau N^{* \prime \prime}(t, \tau u) u_{1}, u_{1}\right) \\
& =\int_{0}^{1} d t \int_{0}^{h(t, u)} d \tau\left(N^{* \prime \prime}(t, \tau u) u_{1}, u_{1}\right)+\int_{0}^{1} d t \int_{h(t, u)}^{1} d \tau\left(N^{* \prime \prime}(t, \tau u) u_{1}, u_{1}\right) \\
& \leq c_{0}\|u\|+\int_{0}^{1} d t\left(\left(B_{1}(t)+\mu I-s I\right) u_{1}, u_{1}\right)
\end{array}
$$

where $h(t, u)=r_{1} /|u(t)|$. Similarly,

$$
\begin{aligned}
\int_{0}^{1} d t\left(\int_{0}^{1} d \tau N^{* \prime \prime}(t, \tau u) u_{2}, u_{2}\right) & \geq \int_{0}^{1} d t \int_{h(t, u)}^{1} d \tau\left(N^{* \prime \prime}(t, \tau u) u_{2}, u_{2}\right) \\
& \geq \int_{0}^{1} d t\left(\left(B_{2}(t)+\mu I+s I\right) u_{2}, u_{2}\right)-c\|u\|
\end{aligned}
$$

for some $c>0$. So by the last three relations, we have

$$
\left(f^{\prime}(u), u_{2}-u_{1}\right) \geq c_{1}\left\|u_{1}\right\|^{2}+c_{2}\left\|u_{2}\right\|^{2}-c_{3}\left(\left\|u_{1}\right\|+\left\|u_{2}\right\|\right) .
$$

Thus for large $R$ with $\left\|u_{1}\right\| \geq R$ or $\left\|u_{2}\right\| \geq R$, we have

$$
\left(-f^{\prime}(u), u_{2}-u_{1}\right)<-1 .
$$

We know from (4-11) that $f$ has no critical point outside $D_{R}$, and that $-f^{\prime}(u)$ points inward to $D_{R}$ on $\partial D_{R}$. So we can define the deformation by negative flow. In fact, for any $u=u_{1}+u_{2} \notin D_{R}$, let $\sigma(\theta, u)=e^{\theta} u_{1}+e^{-\theta} u_{2}$, and $d_{u}=\log \left\|u_{2}\right\|-\log R$. We define the deformation map $\eta:[0,1] \times \mathrm{L} \rightarrow \mathrm{L}$ by

$$
\eta\left(\theta, u_{1}+u_{2}\right)= \begin{cases}u_{1}+u_{2}, & \left\|u_{2}\right\| \leq R \\ \sigma\left(d_{u} \theta, u\right), & \left\|u_{2}\right\|>R .\end{cases}
$$


The map $\eta$ satisfies the properties

$$
\begin{array}{lll}
\eta(0, \cdot)=\mathrm{id}, & \eta(1, \mathrm{~L}) \subset D_{R}, & \eta\left(1, f_{a}\right) \subset D_{R} \cap f_{a} \\
\eta\left(\theta, f_{a}\right) \subset f_{a}, & \left.\eta(\theta, \cdot)\right|_{D_{R}}=\left.i d\right|_{D_{R}} .
\end{array}
$$

Thus the pair $\left(D_{R}, D_{R} \cap f_{a}\right)$ is a deformation retract of the pair $\left(\mathrm{L}, f_{a}\right)$.

Step 3. For large $R,-a>0$, there holds

$$
H_{q}\left(D_{R}, D_{R} \cap f_{a}\right) \cong \delta_{q, r} \mathbb{R} .
$$

In fact, similarly to the above computation, for large $m>0$, we have

$$
\begin{aligned}
& \int_{0}^{1} d t N^{*}(t, u(t)) \\
& \quad=\int_{0}^{1} d t\left(N^{*}(t, 0)+\iint_{[0,1] \times[0,1]} d \tau d s \tau\left(N^{* \prime \prime}(t, \tau s u(t)) u(t), u(t)\right)\right) \\
& \quad \leq \int_{|u(t)| \geq m r_{1}} d t \iint_{[0,1] \times[0,1]} d \tau d s \tau\left(N^{* \prime \prime}(t, \tau s u(t)) u(t), u(t)\right)+c_{m} \\
& \quad \int_{|u(t)| \geq m r_{1} \quad d t \iint_{|s \tau u(t)| \geq r_{1}, \tau, s \in[0,1]} d \tau d s \tau\left(N^{* \prime \prime}(t, \tau s u(t)) u(t), u(t)\right)} d t \quad \iint_{|u(t)| \geq m r_{1}} d \tau d s \tau\left(N^{* \prime \prime}(t, \tau s u(t)) u(t), u(t)\right)+c_{m} \\
& \quad \leq \frac{1}{2} \int_{0}^{1} d t\left(\left(B_{1}(t)+\mu r_{1}, \tau, s \in[0,1]\right.\right.
\end{aligned}
$$

where $c_{m}$ and $k_{m}$ are constants depending only on $m$ and $k_{m} \rightarrow 0$ as $m \rightarrow+\infty$. So for the small $s$ in the step 2 above, we can choose a large number $m$ such that $\int_{0}^{1} d t N^{*}(t, u(t)) \leq \frac{1}{2} \int_{0}^{1} d t\left(\left(B_{1}(t)+\mu I-s I\right)^{-1} u(t), u(t)\right)+C \quad$ for all $u \in \mathrm{L}$ for some constant $C>0$. Thus for any $u=u_{1}+u_{2}$ with $u_{1} \in \mathrm{L}_{\mu}^{-}\left(B_{1}-s I\right)$ and $u_{2} \in \mathrm{L}_{\mu}^{+}\left(B_{2}+s I\right)$ with $\left\|u_{2}\right\| \leq R$, there holds

$$
f(u) \leq-C_{1}\left\|u_{1}\right\|^{2}+C_{2}\left\|u_{1}\right\|+C_{3},
$$

where $C_{j}, j=1,2,3$ are constants and $C_{1}>0$. It implies that $f(u) \rightarrow-\infty$ if and only if $\left\|u_{1}\right\| \rightarrow \infty$ uniformly for $u_{2} \in \mathrm{L}_{\mu}^{+}\left(B_{2}+s I\right)$ with $\left\|u_{2}\right\| \leq R$. In the following we denote by $B_{r}=\{x \in \mathrm{L} \mid\|x\| \leq r\}$ the ball with radius $r$ in $\mathrm{L}$. Therefore for $-a_{1}>-a_{2}$ sufficiently large, there exist three numbers with $R<R_{1}<R_{2}<R_{3}$ 
satisfying

$$
\begin{aligned}
&\left.\left(\mathrm{L}_{\mu}^{+}\left(B_{2}+s I\right) \cap B_{R_{3}}\right) \oplus\left(\mathrm{L}_{\mu}^{-}\right)\left(B_{1}-s I\right) \backslash B_{R_{2}}\right) \subset f_{a_{1}} \cap D_{R_{3}} \\
&\left.\subset\left(\mathrm{L}_{\mu}^{+}\left(B_{2}+s I\right) \cap B_{R_{3}}\right) \oplus\left(\mathrm{L}_{\mu}^{-}\right)\left(B_{1}-s I\right) \backslash B_{R_{1}}\right) \subset f_{a_{2}} \cap D_{R_{3}} .
\end{aligned}
$$

Recall that $\sigma(\theta, u)=e^{\theta} u_{1}+e^{-\theta} u_{2}$. By definition, we have $f(\sigma(0, u))=f(u)>a_{1}$ and $f(\sigma(\theta, u)) \rightarrow-\infty$ as $\theta \rightarrow \infty$ if $u=u_{1}+u_{2} \in D_{R_{3}} \cap\left(f_{a_{2}} \backslash f_{a_{1}}\right)$. It implies that there exists $\theta_{0}=\theta_{0}(u)>0$ such that $f\left(\sigma\left(\theta_{0}, u\right)\right)=a_{1}$. But by $(4-11)$,

$$
\frac{d}{d \theta} f(\sigma(\theta, u)) \leq-1 \quad \text { at any point } \theta>0 .
$$

By the implicit function theorem, $\theta_{0}(u)$ is continuous in $u$. We define another deformation map $\eta_{0}:[0,1] \times f_{a_{2}} \cap D_{R_{3}} \rightarrow f_{a_{2}} \cap D_{R_{3}}$ by

$$
\eta_{0}(\theta, u)= \begin{cases}u & u \in f_{a_{1}} \cap D_{R_{3}}, \\ \sigma\left(\theta_{0}(u) \theta, u\right), & u \in D_{R_{3}} \cap\left(f_{a_{2}} \backslash f_{a_{1}}\right) .\end{cases}
$$

It is clear that $\eta_{0}$ is a deformation from $f_{a_{2}} \cap D_{R_{3}}$ to $f_{a_{1}} \cap D_{R_{3}}$. We now define

$$
\tilde{\eta}(u)=d\left(\eta_{0}(1, u)\right) \quad \text { with } d(u)= \begin{cases}u, & \left\|u_{1}\right\| \geq R_{1}, \\ u_{2}+\frac{u_{1}}{\left\|u_{1}\right\|} R_{1}, & 0<\left\|u_{1}\right\|<R_{1} .\end{cases}
$$

This map defines a strong deformation retract:

$$
\tilde{\eta}: D_{R_{3}} \cap d_{a_{2}} \rightarrow\left(\mathrm{L}_{\mu}^{+}\left(B_{2}+s I\right) \cap B_{R_{3}}\right) \oplus\left(\mathrm{L}_{\mu}^{-}\left(B_{1}-s I\right) \cap\left\{u \in \mathrm{L} \mid\|u\| \geq R_{1}\right\}\right) .
$$

Now we can compute the homology groups

$$
\begin{aligned}
& H_{q}\left(D_{R_{3}}, D_{R_{3}} \cap f_{a_{2}} ; \mathbb{R}\right) \\
& \quad \cong H_{q}\left(D_{R_{3}},\left(\mathrm{~L}_{\mu}^{+}\left(B_{2}+s I\right) \cap B_{R_{3}}\right) \oplus\left(\mathrm{L}_{\mu}^{-}\left(B_{1}-s I\right) \cap\left\{u \in \mathrm{L} \mid\|u\| \geq R_{1}\right\}\right) ; \mathbb{R}\right) \\
& \quad \cong H_{q}\left(\mathrm{~L}_{\mu}^{-}\left(B_{1}-s I\right) \cap B_{R_{3}}, \partial\left(\mathrm{L}_{\mu}^{-}\left(B_{1}-s I\right) \cap B_{R_{3}}\right) ; \mathbb{R}\right) \\
& \quad \cong \delta_{q r} \mathbb{R} .
\end{aligned}
$$

From (4-8), (4-9), and (A.2) below, and by using Equation (4-1), we complete the proof.

Corollary 4.2. Let $H$ satisfy the conditions $\left(H_{0}\right)$ and $\left(H_{\infty}\right)$, and suppose $B_{0}(t)=$ $H^{\prime \prime}(t, 0)$ satisfies one of the twisted conditions:

(i) $B_{1}(t)<B_{0}(t)$, there exists $\lambda \in(0,1)$ such that $v_{L}\left((1-\lambda) B_{1}+\lambda B_{0}\right) \neq 0$;

(ii) $B_{0}(t)<B_{1}(t)$, there exists $\lambda \in(0,1)$ such that $v_{L}\left((1-\lambda) B_{0}+\lambda B_{1}\right) \neq 0$.

Then (1-1) possesses at least one nontrivial solution. Furthermore, if $v_{L}\left(B_{0}\right)=0$ and in (i) we replace the second condition by $\sum_{\lambda \in(0,1)} v\left((1-\lambda) B_{1}+\lambda B_{0}\right) \geq n$, or in (ii) we replace the second condition by $\sum_{\lambda \in(0,1)} v\left((1-\lambda) B_{0}+\lambda B_{1}\right) \geq n$, the Hamiltonian system (1-1) possesses at least two nontrivial solutions. 
Proof. It follows from (2-3), the proof of Theorem 1.1 and (4-2). In the first case, we have $r=i_{L}\left(B_{1}\right) \notin\left[i_{L}\left(B_{0}\right), i_{L}\left(B_{0}\right)+v_{L}\left(B_{0}\right)\right]$. In the second case we have $\left|i_{L}\left(B_{0}\right)-i_{L}\left(B_{1}\right)\right| \geq n$.

The proof of Theorem 1.1 in fact proves this:

Theorem 4.3. Let $H$ satisfy conditions $\left(H_{0}\right)$ and $\left(H_{\infty}\right)$. Suppose $B_{0}(t)=H^{\prime \prime}(t, 0)$ satisfies the twisted conditions

$$
i_{L}\left(B_{1}\right) \notin\left[i_{L}\left(B_{0}\right), i_{L}\left(B_{0}\right)+v_{L}\left(B_{0}\right)\right] .
$$

Then the problem (1-1) possesses at least one nontrivial solution. Moreover, if $v_{L}\left(B_{0}\right)=0$ and $\left|i_{L}\left(B_{1}\right)-i_{L}\left(B_{0}\right)\right| \geq n$, then (1-1) possesses at least two nontrivial solutions.

Remark. The condition $B_{1}(t)<B_{2}(t)$ in Theorem 2.4 can be replaced by $B_{1}(t) \leq$ $B_{2}(t)$ for all $t$ and $B_{2}-B_{1} \geq \delta>0$ for some constant $\delta$ as an operator in L. So the conditions in parts (i) and (ii) of Corollary 4.2 can be replaced by this kind of condition. The condition $J B_{1}(t)=B_{1}(t) J$ in $\left(H_{\infty}\right)$ can be replaced by $J B_{0}(t)=B_{0}(t) J$.

\section{Appendix. Maslov-type index for symplectc paths with Lagrangian boundary} condition

We give a brief introduction to the Maslov-type index for symplectc paths with Lagrangian boundary condition. The details can be found in [Liu 2007]. We denote the symplectic group by

$$
\operatorname{Sp}(2 n)=\left\{M \in \mathrm{L}\left(\mathbb{R}^{2 n}\right) \mid M^{T} J M=J\right\},
$$

and denote the symplectic path space by

$$
\mathrm{P}(2 n)=\left\{\gamma \in C([0,1], \operatorname{Sp}(2 n)) \mid \gamma(0)=I_{2 n}\right\} .
$$

We write a symplectic path $\gamma \in \mathrm{P}(2 n)$, in the form

$$
\gamma(t)=\left(\begin{array}{ll}
S(t) & V(t) \\
T(t) & U(t)
\end{array}\right),
$$

where $S(t), T(t), V(t), U(t)$ are $n \times n$ matrices. The $n$ vectors coming from the rightmost columns of the above matrix are linearly independent and they span a Lagrangian subspace of $\left(\mathbb{R}^{2 n}, \omega_{0}\right)$. In particular, at $t=0$, this Lagrangian subspace is $L_{0}=\{0\} \oplus \mathbb{R}^{n}$.

Definition A.1. We define the $L_{0}$-nullity of any symplectic path $\gamma \in \mathrm{P}(2 n)$ by

$$
v_{L_{0}}(\gamma) \equiv \operatorname{dim} \operatorname{ker}_{L_{0}}(\gamma(1)):=\operatorname{dim} \operatorname{ker} V(1)=n-\operatorname{rank} V(1)
$$


with the $n \times n$ matrix function $V(t)$ defined in (A.1).

We define two subsets of $\mathrm{P}(2 n)$ by

$$
\begin{aligned}
& \mathrm{P}(2 n)_{L_{0}}^{*}=\left\{\gamma \in \mathrm{P}(2 n) \mid v_{L_{0}}(\gamma)=0\right\}, \\
& \mathrm{P}(2 n)_{L_{0}}^{0}=\left\{\gamma \in \mathrm{P}(2 n) \mid v_{L_{0}}(\gamma)>0\right\} .
\end{aligned}
$$

We note that

$$
\operatorname{rank}\left(\begin{array}{c}
V(t) \\
U(t)
\end{array}\right)=n
$$

so the complex matrix $U(t) \pm \sqrt{-1} V(t)$ is invertible. We define a complex matrix function by

$$
\mathrm{Q}(t)=(U(t)-\sqrt{-1} V(t))(U(t)+\sqrt{-1} V(t))^{-1} .
$$

It is easy to see that the matrix $\mathrm{Q}(t)$ is a unitary matrix for any $t \in[0,1]$. We define

$$
M_{+}=\left(\begin{array}{cc}
0 & I_{n} \\
-I_{n} & 0
\end{array}\right), \quad M_{-}=\left(\begin{array}{cc}
0 & J_{n} \\
-J_{n} & 0
\end{array}\right), \quad J_{n}=\operatorname{diag}(-1,1, \ldots, 1) .
$$

For a path $\gamma \in \mathrm{P}(2 n)_{L_{0}}^{*}$, we first adjoin it with a simple symplectic path starting from $J=-M_{+}$, that is, we define a symplectic path by

$$
\tilde{\gamma}(t)= \begin{cases}I \cos (\pi / 2)(1-2 t)+J \sin (\pi / 2)(1-2 t), & t \in[0,1 / 2] \\ \gamma(2 t-1), & t \in[1 / 2,1] .\end{cases}
$$

then we choose a symplectic path $\beta(t)$ in $\operatorname{Sp}(2 n)_{L_{0}}^{*}$ starting from $\gamma(1)$ and ending at $M_{+}$or $M_{-}$. We now define a joint path by

$$
\bar{\gamma}(t)=\beta * \tilde{\gamma}:= \begin{cases}\tilde{\gamma}(2 t), & t \in[0,1 / 2], \\ \beta(2 t-1), & t \in[1 / 2,1] .\end{cases}
$$

By the definition, we see that the symplectic path $\bar{\gamma}$ starting from $-M_{+}$and ending at either $M_{+}$or $M_{-}$. As above, we define

$$
\overline{\mathrm{Q}}(t)=(\bar{U}(t)-\sqrt{-1} \bar{V}(t))(\bar{U}(t)+\sqrt{-1} \bar{V}(t))^{-1} .
$$

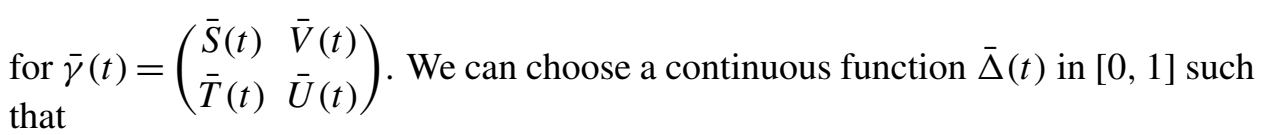

$$
\operatorname{det} \overline{\mathrm{Q}}(t)=e^{2 \sqrt{-1} \bar{\Delta}(t)}
$$

By the above arguments, we see that the number $\frac{1}{\pi}(\bar{\Delta}(1)-\bar{\Delta}(0)) \in \mathbb{Z}$ and it does not depend on the choice of the function $\bar{\Delta}(t)$. 
Definition A.2. For a symplectic path $\gamma \in \mathrm{P}(2 n)_{L_{0}}^{*}$, we define the $L_{0}$-index of $\gamma$ by

$$
i_{L_{0}}(\gamma)=\frac{1}{\pi}(\bar{\Delta}(1)-\bar{\Delta}(0)) .
$$

Definition A.3. For a symplectic path $\gamma \in \mathrm{P}(2 n)_{L_{0}}^{0}$, we define the $L_{0}$-index of $\gamma$ by

$$
i_{L_{0}}(\gamma)=\inf \left\{i_{L_{0}}(\tilde{\gamma}) \mid \tilde{\gamma} \in \mathrm{P}(2 n)_{L_{0}}^{*} \text {, and } \tilde{\gamma} \text { is sufficiently close to } \gamma\right\} .
$$

We note that $\Lambda(n)=U(n) / O(n)$; this means that for any linear subspace $L \in$ $\Lambda(n)$, there is an orthogonal symplectic matrix

$$
P=\left(\begin{array}{cc}
A & -B \\
B & A
\end{array}\right)
$$

with $A \pm \sqrt{-1} B \in U(n)$ such that $P L_{0}=L$. $P$ is uniquely determined by $L$ up to an orthogonal matrix $C \in O(n)$. It means that for any other choice $P^{\prime}$ satisfying above conditions, there exists a matrix $C \in O(n)$ such that

$$
P^{\prime}=P\left(\begin{array}{ll}
C & 0 \\
0 & C
\end{array}\right) \text {. }
$$

See [McDuff and Salamon 1998, Lemma 2.31]. We define the conjugated symplectic path $\gamma_{c} \in \mathrm{P}(2 n)$ of $\gamma$ by $\gamma_{c}(t)=P^{-1} \gamma(t) P$.

Definition A.4. We define the $L$-nullity of any symplectic path $\gamma \in \mathrm{P}(2 n)$ by

$$
v_{L}(\gamma) \equiv \operatorname{dim} \operatorname{ker}_{L}(\gamma(1)):=\operatorname{dim} \operatorname{ker} V_{c}(1)=n-\operatorname{rank} V_{c}(1),
$$

The $n \times n$ matrix function $V_{c}(t)$ is defined in (A.1) with the symplectic path $\gamma$ replaced by $\gamma_{c}$, that is,

$$
\gamma_{c}(t)=\left(\begin{array}{ll}
S_{c}(t) & V_{c}(t) \\
T_{c}(t) & U_{c}(t)
\end{array}\right) .
$$

Definition A.5. For a symplectic path $\gamma \in \mathrm{P}(2 n)$, we define the $L$-index of $\gamma$ by

$$
i_{L}(\gamma)=i_{L_{0}}\left(\gamma_{c}\right)
$$

Theorem A.6. If $\gamma \in \mathrm{P}(2 n)_{L}^{0}$, there is a family of paths $\gamma_{s} \in \mathrm{P}(2 n)_{L}$ depend continuous on $s \in[-1,1]$ such that $\gamma_{0}=\gamma, \gamma_{s} \in \mathrm{P}(2 n)_{L}^{*}, s \neq 0$ and

$$
i_{L}\left(\gamma_{s}\right)-i_{L}\left(\gamma_{-s}\right)=v_{L}(\gamma) \quad \text { for all } s \in(0,1],
$$

and

$$
i_{L}(\gamma)=i_{L}\left(\gamma_{-s}\right), s \in(0,1]
$$


For a symmetric matrix function $B:[0,1] \rightarrow \mathrm{L}_{s}(2 n)$, we consider the functional

$$
f(z)=\int_{0}^{1}\left(\frac{1}{2}(-J \dot{z}, z)-(B(t) z, z)\right) d t, \quad z \in W_{L},
$$

where $W_{L}=\left\{z=(x, y)^{T} \in W^{1,2}\left([0,1], \mathbb{R}^{2 n}\right) \mid z(0), z(1) \in L\right\} \subset L^{2}$. By the saddle point reduction methods (see [Amann 1979; Amann and Zehnder 1980; Long 1993; 2002; Liu 2007]), there exists a finite-dimensional subspace $X$ of $W_{L}$ with $\operatorname{dim} X=2 d+n$ and an injection map $X \rightarrow W_{L}$, such that the function $a(x)=f(u(x))$ is $C^{2}$ and we have:

Theorem A.7. For any $L \in \Lambda(n)$,

$$
\begin{aligned}
& m^{-}(a)=d+i_{L}(B)+n, \\
& m^{0}(a)=v_{L}(B), \\
& m^{+}(a)=d-i_{L}(B)-v_{L}(B),
\end{aligned}
$$

where $m^{*}(a)$ for $*=+, 0,-$ are respectively the positive, null and the negative Morse indices of the function $a(x)$ at the origin.

Theorem A.8. For any symplectic path $\gamma \in \mathrm{P}(2 n)$, there holds

$$
i_{L_{0}}(\gamma)=\sum_{j=1}^{n} E\left(\frac{\theta_{j}(1)-\theta_{j}(0)}{2 \pi}\right),
$$

where $E(a)=\max \{k \in \mathbb{Z} \mid k<a\}$ and $\lambda_{j}(t)=e^{\sqrt{-1} \theta_{j}(t)}$ are the eigenvalues of $\mathrm{Q}(t)$ defined in (A.3).

\section{Acknowledgement}

A part of this paper was done while the author visiting Chern Institute of Mathematics, and the author would like to thank the Institute for partial support. The author appreciates the referee for his/her useful revision suggestions and for correcting some mistakes.

\section{References}

[Amann 1979] H. Amann, "Saddle points and multiple solutions of differential equations", Math. Z. 169:2 (1979), 127-166. MR 80j:47078 Zbl 0414.47042

[Amann and Zehnder 1980] H. Amann and E. Zehnder, "Nontrivial solutions for a class of nonresonance problems and applications to nonlinear differential equations", Ann. Scuola Norm. Sup. Pisa Cl. Sci. (4) 7:4 (1980), 539-603. MR 82b:47077 Zbl 0452.47077

[Arnold 1986] V. I. Arnold, "The first steps of symplectic topology", Uspekhi Mat. Nauk 41:6(252) (1986), 3-18, 229. MR 89d:58034 Zbl 0618.58021

[Bott 1982] R. Bott, "Lectures on Morse theory, old and new", Bull. Amer. Math. Soc. (N.S.) 7:2 (1982), 331-358. MR 84m:58026a Zbl 0505.58001 
[Chang 1981] K. C. Chang, "Solutions of asymptotically linear operator equations via Morse theory”, Comm. Pure Appl. Math. 34:5 (1981), 693-712. MR 82m:58015 Zbl 0444.58008

[Chekanov 1996] Y. V. Chekanov, "Hofer's symplectic energy and Lagrangian intersections", pp. 296-306 in Contact and symplectic geometry (Cambridge, 1994), edited by C. B. Thomas, Publ. Newton Inst. 8, Cambridge Univ. Press, Cambridge, 1996. MR 98b:58058 Zbl 0867.58026

[Chekanov 1998] Y. V. Chekanov, "Lagrangian intersections, symplectic energy, and areas of holomorphic curves”, Duke Math. J. 95:1 (1998), 213-226. MR 99k:58034 Zbl 0977.53077

[Clarke 1978] F. H. Clarke, "Solution périodique des équations hamiltoniennes", C. R. Acad. Sci. Paris 287 (1978), 951-952. Zbl 0422.35005

[Clarke 1979] F. H. Clarke, "A classical variational principle for periodic Hamiltonian trajectories", Proc. Amer. Math. Soc. 76:1 (1979), 186-188. MR 84h:34071 Zbl 0434.70022

[Clarke 1981] F. H. Clarke, "Periodic solutions to Hamiltonian inclusions", J. Differential Equations 40:1 (1981), 1-6. MR 83a:58035 Zbl 0461.34030

[Clarke and Ekeland 1978] F. H. Clarke and I. Ekeland, "Solutions périodiques, de période donnée, des équations hamiltoniennes”, C. R. Acad. Sci. Paris Sér. A-B 287:15 (1978), A1013-A1015. MR 80b:49025 Zbl 0404.34033

[Clarke and Ekeland 1980] F. H. Clarke and I. Ekeland, "Hamiltonian trajectories having prescribed minimal period”, Comm. Pure Appl. Math. 33:2 (1980), 103-116. MR 81e:70017 Zbl 0403.70016

[Clarke and Ekeland 1982] F. H. Clarke and I. Ekeland, "Nonlinear oscillations and boundary value problems for Hamiltonian systems”, Arch. Rat. Mech. Anal. 78:4 (1982), 315-333. MR 83h:58038 Zbl 0514.34032

[Conley and Zehnder 1984] C. Conley and E. Zehnder, "Morse-type index theory for flows and periodic solutions for Hamiltonian equations", Comm. Pure Appl. Math. 37:2 (1984), 207-253. MR 86b:58021 Zbl 0559.58019

[Dong 2005] Y. Dong, "Maslov type index theory for linear Hamiltonian systems with Bolza boundary value conditions and multiple solutions for nonlinear Hamiltonian systems", Pacific J. Math. 221:2 (2005), 253-280. MR 2006k:37163 Zbl 05031482

[Ekeland 1990] I. Ekeland, Convexity methods in Hamiltonian mechanics, vol. 19, Ergebnisse der Mathematik, Springer, Berlin, 1990. MR 91f:58027 Zbl 0707.70003

[Fei and Qiu 1996] G. Fei and Q. Qiu, "Minimal period solutions of nonlinear Hamiltonian systems", Nonlinear Anal. 27:7 (1996), 821-839. MR 97i:58143 Zbl 0858.34034

[Floer 1988] A. Floer, "Morse theory for Lagrangian intersections", J. Differential Geom. 28:3 (1988), 513-547. MR 90f:58058 Zbl 0674.57027

[Floer 1989] A. Floer, "Cuplength estimates on Lagrangian intersections", Comm. Pure Appl. Math. 42:4 (1989), 335-356. MR 90g:58034 Zbl 0683.58017

[Girardi and Matzeu 1991] M. Girardi and M. Matzeu, "Dual Morse index estimates for periodic solutions of Hamiltonian systems in some nonconvex superquadratic cases", Nonlinear Anal. 17:5 (1991), 481-497. MR 92k:58224 Zbl 0779.34033

[Guo and Liu 2007] F. Guo and C. Liu, "Multiplicity of Lagrangian orbits on symmetric star-shaped hypersurfaces", Nonlinear Anal. (2007).

[Hofer 1988] H. Hofer, "Lusternik-Schnirelman-theory for Lagrangian intersections", Ann. Inst. H. Poincaré Anal. Non Linéaire 5:5 (1988), 465-499. MR 91b:58068 Zbl 0669.58006

[Liu 2001] C. G. Liu, "Dual Morse index for subquadratic Hamiltonian systems and the stability of the closed characteristics", Acta Math. Sinica (Chin. Ser.) 44 (2001), 1073-1088. MR 2002j:37090 Zbl 1012.37047 
[Liu 2005a] C.-G. Liu, "Cup-length estimate for Lagrangian intersections", J. Differential Equations 209:1 (2005), 57-76. MR 2005g:53176 Zbl 02136639

[Liu 2005b] C.-G. Liu, "A note on the monotonicity of the Maslov-type index of linear Hamiltonian systems with applications", Proc. Roy. Soc. Edinburgh Sect. A 135:6 (2005), 1263-1277. MR 2007d:53135 Zbl 05001218

[Liu 2007] C. Liu, "Maslov-type index theory for symplectic paths with Lagrangian boundary conditions", Advanced Nonlinear Studies 7:1 (2007), 131-161.

[Long 1993] Y. Long, Index Theory of Hamiltonian Systems with Applications, Science Press, Beijing, 1993. In Chinese.

[Long 2002] Y. Long, Index theory for symplectic paths with applications, Progress in Mathematics 207, Birkhäuser Verlag, Basel, 2002. MR 2003d:37091 Zbl 1099.81543

[McDuff and Salamon 1998] D. McDuff and D. Salamon, Introduction to symplectic topology, Second ed., Oxford Mathematical Monographs, The Clarendon Press Oxford University Press, New York, 1998. MR 2000g:53098 Zbl 1066.53137

[Mohnke 2001] K. Mohnke, "Holomorphic disks and the chord conjecture", Ann. of Math. (2) 154:1 (2001), 219-222. MR 2002f:53147 Zbl 1007.53065

[Oh 1995] Y.-G. Oh, "Floer cohomology of Lagrangian intersections and pseudo-holomorphic disks. III. Arnold-Givental conjecture", pp. 555-573 in The Floer memorial volume, edited by $\mathrm{H}$. Hofer et al., Progr. Math. 133, Birkhäuser, Basel, 1995. MR 96k:58037 Zbl 0842.58033

[Ono 1996] K. Ono, "Lagrangian intersection under Legendrian deformations", Duke Math. J. 85:1 (1996), 209-225. MR 97g:58036 Zbl 0868.58035

Received June 6, 2006. Revised October 14, 2006.

\section{Chun-Gen LiU}

SCHOOL OF MATHEMATICS AND LPMC

NANKAI UNIVERSITY

TIANJIN 300071

CHINA

liucg@nankai.edu.cn 